\title{
CORRELATION BETWEEN TIME TO POSITIVITY BLOOD CULTURE AND PROCALCITONIN IN BACTEREMIA PATIENTS
}

\author{
Nelly Elfrida Samosir, Ricke Loesnihari, Adi Koesoema Aman \\ Department of Clinical Pathology, Faculty of Medicine, University of North Sumatra/Installation of Clinical Pathology of Adam Malik \\ Hospital, Medan, Indonesia. E-mail: clinpathusu@yahoo.com
}

\begin{abstract}
Bacteremia causes a high mortality rate. Early detection of bacteremia is very important. The gold standard for bacteremia is blood culture, which takes between $24-48$ hours. Procalcitonin (PCT) is a marker of infection caused by bacteria that can be detected quickly in 2-6 hours. Time to Positivity (TTP) of blood culture is affected by the initial amount of bacteria and the addition of PCT stimulated by bacteria causing bacteremia where short TTP and high PCT show bad clinical conditions. This study aimed to investigate the correlation of TTP of blood cultures with PCT levels in bacteremia patients. The study design was cross-sectional, conducted on 46 bacteremia patients who met the inclusion and exclusion criteria, in patients with bacteremia. Time to positivity was calculated by Bactec 9050 , and miniVIDAS B.R.A.H.M.S. analyzed level of PCT. Laboratory examinations were conducted in the Department of Clinical Pathology, Faculty of Medicine, North Sumatera University/Installation of Clinical Pathology of Adam Malik Hospital, Medan, during June - October 2016. There was a significant correlation between TTP blood culture and PCT in bacteremia patients $(p<0.05)$ with $r-0.294$. There was no significant correlation between TTP and PCT in bacteremia, which was caused by Gram-positive bacteria or Gram-negative bacteria ( $p>0.05$ ). Procalcitonin was significantly higher in bacteremia, which was caused by Gram-negative bacteria compared to Gram-positive bacteria $(p<0.05)$. There was a significant correlation between TTP blood culture and PCT on bacteremia patients. Significantly higher levels of PCT in cases of bacteremia are more likely to be caused by Gram-negative bacteria than Gram-positive bacteria.
\end{abstract}

Key words: Time to positivity, blood culture, procalcitonin, bacteremia

\section{INTRODUCTION}

Bacteremia cases cause high morbidity and mortality. For immediate handling of the patient, the detection of bacteremia needs to be as short as possible. Although blood culture is the gold standard for detecting infections in the bloodstream, the results can only be obtained after 24 to 48 hours or more, which may lead to the delay of the patient's treatment. ${ }^{1,2}$

Time to Positivity (TTP) blood culture is the starting time of the sample incubated in the instrument until the alarm sign of germ growth. The bacterial load of blood culture is often assessed by the TTP and influenced by the concentration of pathogens in the primary sample and pathogenic species. ${ }^{3}$ In addition to the initial inoculum, other factors affecting TTP are the volume of blood inserted into the culture bottle and the incubation conditions, collection time, processing parameters and delay of sample transfer. ${ }^{3,4}$

Some reports show that a short TTP is associated with poor clinical outcome and death, and may be used as an independent prognostic instrument in bacteremia caused by Pseudomonas aeruginosa, Klebsiella pneumonia, Escherichia coli, and as a surrogate marker for the output of bacteremia caused by Streptococcus pneumonia. ${ }^{4-9}$

Procalcitonin (PCT) is a prohormone of calcitonin synthesized by the thyroid gland cell, a functional protein consisting of 114-116 amino acids, which is found in high levels strongly associated with systemic bacterial infections and disease severity, first performed by Assicot et al. who differentiated bacterial meningitis and viral meningitis. ${ }^{10,11}$ After adequate stimulus PCT can be detected in the circulation within 2-6 hours. ${ }^{12}$ Procalcitonin levels correlate with the severity of the bacterial infection and bacterial count. High PCT levels have a strong positive predictive value of severe sepsis and septic shock. ${ }^{11}$

From the studies above in which a short TTP is associated with high morbidity and mortality and high PCT is associated with the severe clinical outcome on bacteremia. This study aimed to investigate the correlation of TTP of blood cultures 
with PCT levels in bacteremia patients.

\section{METHODS}

This study was a cross-sectional study conducted in the Department of Clinical Pathology, Faculty of Medicine, Adam Malik Hospital from June to October 2016. The study population was patients with suspected bacteremia hospitalized in the Adam Malik Hospital, with inclusion criteria of two or more of the following: temperature $<36^{\circ} \mathrm{C}$ or $>38^{\circ} \mathrm{C}$, leukocytes $<4000 / \mathrm{mm}^{3}$ or $>12.000 / \mathrm{mm}^{3}$, tachypnea RR $>24 x /$ minute, HR tachycardia $>90 x /$ min, or hypotension. Patients with burns, thyroid carcinoma, polymicrobial growth, fungi, bacterial contaminants (coagulase negative Staphylococci with TTP $>24 \mathrm{~h}$, Bacillus spp. Diphtheroid) were excluded from this study. The sample of the research was the time when blood culture was performed, and growth of monomicrobial bacteria was shown.

Aseptic sampling was performed using $\mathrm{CHG} \mathrm{0.5 \%}$ swab alcohol (70\% isopropyl alcohol, 0.5\% chlorhexidine gluconate) for blood culture. Blood cultures were performed by inoculating/incubating $10 \mathrm{~mL}$ of blood into a BD Bactec Aerobic Plus bottle and incubated in the BACTEC 9050 immediately after sampling. The BACTEC instrument recording the time of the bottle was inserted, and the timing of flagging, the start time of the sample incubated until the appearance of flagging was recorded as TTP. The identification was done by Gram staining, followed by inoculation on a solid medium of $5 \%$ sheep blood and MacConkey. Identification was done by automated BD Phoenix system.

A miniVIDAS B.R.A.H.M.S analyzer performed procalcitonin level examination, according to the instructions in the kit. The lowest detection result was $0.05 \mathrm{ng} / \mathrm{mL}$, and results $>200 \mathrm{ng} / \mathrm{mL}$ were diluted with the serum of patients with normal PCT levels.

The Kolmogorov-Smirnov normality test was used to see the normality of the data, non-normally distributed quantitative variables were reported as median with their range, and their differences were evaluated with Mann-Whitney test, while normally distributed variables were reported as a mean \pm SD and their differences were evaluated with the t-independent test. Correlation of variables was evaluated by Spearman correlation test in nonnormally distributed and Pearson correlation test in normally distributed and $\mathrm{p}<0.05$ was considered significant.

Ethical Clearance was obtained from the ethical commission of Adam Malik Hospital, Faculty of
Medicine, the University of North Sumatra with number 410/TGL/KEPK FK USU-RSUP HAM/2016.

\section{RESULTS AND DISCUSSION}

A total of 229 patients with suspected bacteremia of which blood cultures were performed. The results were 58 samples (25.32\%) with positive cultures and 171 samples (74.67\%) negative. From the 58 samples with positive cultures, 46 (79.31\%) were monomicrobial bacteria, 7 (12.07\%) fungi, 3 (5.17\%) contaminant bacteria (coagulase negative Staphylococci with TTP >24h, Bacillus spp. Diphtheroid), and 2 (3.45\%) polymicrobial bacteria were found.

A total of 46 samples with monomicrobial growth were used as the samples for this study, which consisted of $21(45.7 \%)$ males, $25(54.3 \%)$ females. The age characteristics of this study were in the range of 20-78 years, Gram-positive bacteria were 26 (56.5\%), and Gram-negative bacteria were 20 (43.5\%) (Table 1).

The median TTP was 17.58 (range 6.33-58.83) hours, median PCT 7.47 (range 0.15 - 487.2) ng/mL, based on Gram staining as Gram-positive median 3.65 (range 0.15-65.58) ng/mL and Gram-negative 11.54 (range 0.46-487.2) ng/mL (Table 2).

The mean TTP of Gram-positive, Gram-negative, coagulase-positive Staphylococci, coagulase-negative Staphylococci, respectively, were 20.10 \pm 7.03 , $19.62 \pm 13.88,20.67 \pm 9.84,19.98 \pm 6.05$ hours (Table 3 ). The mean PCT of positive-coagulase Staphylococci and negative-coagulase Staphylococci respectively, were $11.75 \pm 21.64,9.91 \pm 17.47 \mathrm{ng} / \mathrm{mL}$ (Table 4).

The positivity of blood cultures in this study was $25.32 \%$. Another researcher found the same result with a quite low positivity ranging between 7.6\% - 19.5\%. ${ }^{1,2,13-15}$ The low positivity rate in this study was probably caused by the amount of culture taken with only one bottle of $10 \mathrm{~mL}$ blood volume, and regardless of the history of antibiotic use, where on the Adam Malik Hospital is a tertiary referral hospital, in which the likelihood of patients already receiving treatment with antibiotics previously was quite large. In a study conducted by Dolma et al. who collected two bottles, there was a positive increase in the second bottle wherein the first bottle was $37 \%$ positivity but $63 \%$ in the second bottle. ${ }^{16}$

Three of the most common causes of bacteremia were Staphylococcus epidermidis, Staphylococcus aureus, and Escherichia coli (Table 1). The cause of bacteremia by Gram-based staining revealed a result of Gram-positive 26 (56.5\%) and Gram-negative 20 
Table 1. Bacteremia microbes

\begin{tabular}{|c|c|c|}
\hline No & Microbe & Number \\
\hline & Gram-positive & \\
\hline 1 & Staphylococcus epidermidis & 8 \\
\hline 2 & Staphylococcus aureus & 7 \\
\hline 3 & Staphylococcus hominis & 4 \\
\hline 4 & Staphylococcus haemolyticus & 4 \\
\hline 5 & Streptococcus pyogenes & 2 \\
\hline \multirow[t]{3}{*}{6} & Enterococcus faecalis & 1 \\
\hline & & Total 26 \\
\hline & Gram-negative & \\
\hline 7 & Escherichia coli & 6 \\
\hline 8 & Acinetobacter baumannii & 3 \\
\hline 9 & Burkholderia cepacea & 2 \\
\hline 10 & Klebsiella pneumoniae ssp. pneumonia & 2 \\
\hline 11 & Pseudomonas aeruginosa & 1 \\
\hline 12 & Shigella flexneri & 1 \\
\hline 13 & Acinetobacter sp. & 1 \\
\hline 14 & Enterobacter cloacae & 1 \\
\hline 15 & Citrobacter farmer & 1 \\
\hline 16 & Salmonella typhi & 1 \\
\hline \multirow[t]{3}{*}{17} & Serratia plymuthica & 1 \\
\hline & & Total 20 \\
\hline & Total & 46 \\
\hline
\end{tabular}

Table 2. Time to positivity blood culture and procalcitonin level characteristics

\begin{tabular}{lcccccccc}
\hline \multicolumn{1}{c}{ Variable } & N & Mean & Median & SD & Min & Max & $\begin{array}{c}\text { Normality } \\
\text { test } \\
\text { (p-value) }\end{array}$ & Normality \\
\hline TTP (hour) & 46 & & 17.58 & & 6.33 & 58.83 & 0.019 & Abnormal \\
PCT (ng/mL) & 46 & & 7.47 & & 0.15 & 487.2 & $<0.001$ & Abnormal \\
TTP (hour) & & & & & & & & \\
$\quad$ Gram-positive & 26 & 20.10 & & 7.03 & 11.83 & 42 & 0.099 & Normal \\
$\quad$ Gram-negative & 20 & 19.62 & & 13.88 & 6.33 & 58.83 & 0.364 & Normal \\
PCT (ng/mL) & & & & & & & & \\
$\quad$ Gram-positive & 26 & & 3.65 & & 0.15 & 65.58 & 0.018 & Abnormal \\
$\quad$ Gram-negative & 20 & & 11.54 & & 0.46 & 487.2 & 0.009 & Abnormal \\
TTP (hour) & & & & & & & & \\
$\quad$ Coagulase positive & 7 & 20.67 & & 9.84 & 11.83 & 42.0 & 0.356 & Normal \\
$\quad$ Coagulase negative & 16 & 19.98 & & 6.05 & 13.67 & 40.0 & 0.296 & Normal \\
PCT (ng/mL) & & & & & & & & \\
$\quad$ Coagulase positive & 7 & 11.75 & & 21.64 & 0.15 & 60.24 & 0.168 & Normal \\
$\quad$ Coagulase negative & 16 & 9.91 & & 17.47 & 0.17 & 65.58 & 0.96 & Normal \\
\hline
\end{tabular}

(43.5\%). The same result was found by Gopi et al. and Abe et al. studies. ${ }^{13,17}$ The results was different from those found by Guo et al., in which Gram-negative was higher than Gram-positive. ${ }^{11}$ The most common cause of sepsis was Gram-negative bacteria, but currently, the most common cause of sepsis is Gram-positive bacteria due to the increase of multi-drug resistant bacteria. $^{17}$

The Staphylococcus group caused fifty percent (50\%) of bacteremia cases in this study, with 16 (34.78\%) cases caused by coagulase-negative Staphylococci. The finding showed no difference of 
Table 3. Time to positivity, and PCT comparisons in bacteremia caused by Gram-positive bacteria and Gram-negative bacteria

\begin{tabular}{|c|c|c|c|}
\hline Variable & $n$ & $\bar{x} \pm S D$ & $p$. \\
\hline \multicolumn{4}{|l|}{ TTP (hour) ${ }^{\text {a) }}$} \\
\hline Gram-positive & 26 & $20.11 \pm 7.04$ & 0.888 \\
\hline Gram-negative & 20 & $\begin{array}{c}19.63 \pm 13.88 \\
\text { Median }\end{array}$ & \\
\hline \multicolumn{4}{|l|}{ PCT $(n g / m L)^{b)}$} \\
\hline Gram-positive & 26 & 3.65 & $0.007^{*}$ \\
\hline Gram-negative & 20 & 11.54 & \\
\hline
\end{tabular}

Notes: a) t-independent test, b) Mann-Whitney test, * Significant

Table 4. Correlation between TTP and PCT in bacteremia patient

\begin{tabular}{lccc}
\hline Correlation between variables & $n$ & $r$ & $p$. \\
\hline TTP with PCT & 46 & -0.294 & $0.047^{\star}$ \\
TTP with PCT Gram-positive & 26 & -0.292 & 0.148 \\
TTP with PCT Gram-negative & 20 & -0.206 & 0.348 \\
\hline
\end{tabular}

TTP and PCT between coagulase-positive Staphylococci and coagulase-negative Staphylococci with consecutive $p=0.839$ and $p=0.830$ and by using Pearson correlation test. There was no relation between TTP and PCT neither on coagulase positive Staphylococci $\mathrm{p}=0.679$ nor coagulase negative Staphylococci with $\mathrm{p}=0.736$ (data not shown). Although the most common contaminant microbe was from the coagulase-negative Staphylococci group, many studies have shown that this bacteria group was the most common cause of infections in people with a low immune system and patients with intravascular and implant usage..$^{15,18-20}$ The virulence mechanism of coagulase-negative Staphylococci was less clearly known. Colonization of the polymer surface of medical equipment through the formation of biofilms in layers was alleged to be an important factor. ${ }^{19}$ To determine whether coagulase-negative Staphylococci was the cause of bacteremia was difficult if the number of cultures performed used only one bottle, so some previous studies used TTP to differentiate bacteria from Gram-positive and contaminant groups by looking at TTP. Some researchers, including McGowan et al., concluded that Gram-positive bacterial blood culture detection was faster than Gram-positive contaminant bacteria (15.5 hours vs. 25.0 hours). ${ }^{21}$ Ruimy et al., obtained a median of 14-hours TTP for S.aureus while 26-hours for coagulase-negative Staphylococci. TTP of 2 - 9 hours had $83.3 \%$ probability that the growing bacteria was S.aureus, and vice versa if TTP was $\geq 18 \mathrm{~h}$, a $90.8 \%$ chance of the growth was coagulase-negative Staphylococci. ${ }^{22} \mathrm{~A}$ prior research by Garcia-Vazquez et al., showed that bacteremia caused by coagulase-negative Staphylococci with TTP of $<16$ hours was strongly suspected bacteremia. ${ }^{23}$ Kassis et al. claimed that to distinguish coagulase-negative Staphylococci as a cause of bacteremia or just as a contaminant was when coagulase-negative Staphylococci with TTP > 20 hours. $^{24}$

Coagulase negative Staphylococci with TTP > 24 hours excluded from the study as being considered bacterial contaminants. Statistical analysis showed no significant differences in both TTP and PCT between coagulase-positive Staphylococci and coagulase-negative Staphylococci.

In this study, there was no significant difference between Gram-positive and Gram-negative TTP as the mean TTP for Gram-positive was $20.11 \pm 7.04$ hours, and Gram-negative was $19.63 \pm 13.88$ hours (Table 3). These results were consistent with the results of a study conducted by Gopi et al. in which the mean TTP value of Gram-positive bacteria was 19.33 hours, and Gram-negative was 19.06 hours. Different results were obtained by Samir et al, with a mean TTP for Gram-negative rods was 12.8 hours while for Gram-positive was 19.0 hours. ${ }^{13,14}$ This difference was thought to be influenced by several factors, including the number of bacteria, species of microorganisms, blood volume inserted into the culture bottle, the source of infection, previous antibiotic treatment and clinical characteristics of the 
patient. Species of microorganisms were also one important factor that affected bacterial growth time. ${ }^{6}$

In this study, the researchers found that the levels of PCT produced by Gram-negative bacterial infections were significantly higher than those by Gram-positive bacterial infection with $p<0.05$ (Table 3). Procalcitonin ability to differentiate infections by Gram-positive and Gram-negative bacteria was found in several previous studies where PCT levels were significantly higher in bacteremia caused by Gram-negative bacterial infections compared to Gram-positive. ${ }^{11,25-28}$ Differences in PCT production were due to differences in interactions between Gram-positive and Gram-negative bacteria with the host cells, involving lipoteichoic, peptidoglycan and Lipopolysaccharide (LPS) acids and Pathogen-Associated Molecular Pattern (PAMPs) differences, involving different TLRs, expressed in human cells. Gram-positive bacteria will activate the TLR2 pathway, whereas Gram-negative bacteria activate the TLR4 pathway which will result in differences in cytokine production such as interleukin-1 $\beta$, interleukin-6 (IL-6), and $\alpha$-factor necrosis factor, which ultimately stimulates transcription of calcitonin- mRNA and release PCT from various body tissues. ${ }^{11,25,29}$ Gram-negative bacteria can produce endotoxins in which this endotoxin may also be released when the cell dies, which causes PCT levels to remain high. In bacteremia patients, PCT levels also differ according to species of the bacteria. ${ }^{30}$

The results of this study showed three samples with very high PCT levels where the cultures were Gram-negative bacteria from the Enterobacteriaceae group, Escherichia coli, Shigella flexneri, and Citrobacter farmeri with PCT levels of $487.2 \mathrm{ng} / \mathrm{mL}, 482.2 \mathrm{ng} / \mathrm{mL}$ and $252.8 \mathrm{ng} / \mathrm{mL}$, respectively. This was in line with previous studies in which higher levels of PCT were attributed to Enterobacteriaceae gram-negative bacteria compared with the non-fermenter Gram-negative bacteria. ${ }^{25,30}$ Watanabe et al. in his research found that ESBL bacteria positive resulted in higher PCT levels than ESBL negative bacteria. ${ }^{27}$ In this study, Escherichia coli bacteria with ESBL positive resulted in a wide variation in PCT levels, one with a very high PCT level of $487.2 \mathrm{ng} / \mathrm{mL}$ and one with levels of 0.73 $\mathrm{ng} / \mathrm{mL}$ and no appropriate reason was found to explain these findings.

The correlation between TTP and PCT was tested by Spearman correlation test. There was a significant correlation $(p<0.05)$ between TTP and PCT, with $r=-0.294$. The correlation value described the inverse relationship, meaning that the smaller TTP was, the bigger $\mathrm{PCT}$, but the correlation was weak
(Table 4). This was following the results of research conducted by Hattori et al., and Nieuwkoop et al. where PCT accurately predicted bacterial counts and the presence of bacteremia in urinary tract infection with fever patients. ${ }^{2,31}$ In the group of bacteria divided by Gram staining, neither Gram-positive nor Gram-negative bacteria showed a significant relationship between TTP and PCT, in which the result for Gram-positive was $p=0.148$ with $r=-0.292$ and Gram-negative was $p=0.348$ with $r=-0.206$ with a weak correlation (Table 4).

The results of this study on the relationship between TTP and PCT indirectly reinforced the notion that PCT examinations could be used to distinguish between contamination and actual bloodstream infections by coagulase-negative Staphylococci. ${ }^{32}$ Given the initial number of bacteria in the inoculum affecting the TTP value. The short TTP value described the number of more pathogens in blood culture samples, as well as some studies showing that shorter TTP values were associated with a higher risk of death at bacteremia patients. Researchers concluded that the shorter the value of TTP, the more bacteria that would stimulate the production of PCT. The relationship between TTP and PCT could support the idea that TTP values could be used to predict bacteremia severity. 3,6

Limitations of this study were samples for blood cultures taken used only one bottle of $10 \mathrm{~mL}$ volume, due to the difficulty of obtaining consent from the patient. Previous antibiotic usage history could not be traced where the possibility of antibiotics before admission to the hospital was considerable.

\section{CONCLUSION AND SUGGESTION}

There was a significant relationship between TTP blood cultures with PCT levels in patients with bacteremia with negative correlation. This relationship showed that the shorter value of TTP, the greater the PCT level will be. Significantly higher levels of PCT in cases of bacteremia were more likely to be caused by Gram-negative bacteria than Gram-positive bacteria. Researchers suggest that further research should be conducted on the correlation between TTP with the level of PCT based on the species of the bacteria.

\section{REFERENCES}

1. Oussalah A, Janina f, Pierre FT, Pharm D, Nejla A, Isabelle $A G$, et al. Diagnostic accuracy of procalcitonin for predicting blood culture results in patients with 
suspected bloodstream infection. Medicine, 2015; 94(44): e1774

2. Hattori T, Nishiyama H, Kato H, Ikegami S, Nagayama $\mathrm{M}$, Asami $\mathrm{S}$, et al. Clinical value of procalcitonin for patients with suspected bloodstream infection. Am J Clin Pathol, 2014; 141: 43-51.

3. Ning Y, Hu R, Yao G, Bo S. Time to positivity of blood culture and its prognostic value in the bloodstream. 2016; 35(4): 619-24

4. Willmann M, Kuebart I, Vogel W, Flesch I, Market U, Marschal $M$, et al. Time to positivity as a prognostic tool in patients with pseudomonas aeruginosa bloodstream infection. Journal of Infection, 2013; 67(5): 416-23.

5. Hsu M, Huang $Y$, Hsu $H$, Liao $C$. Sequential time to positivity of blood cultures can be a predictor of prognosis of patients with persistent Staphylococcus aureus bacteremia. Clin Microbiol Infect, 2014; 20:892-8.

6. Tang PC, Lee CC, Li C, Li M, Ko W, Lee N. Time-to-positivity of blood culture: An independent prognostic factor of monomicrobial Pseudomonas aeruginosa bacteremia, Journal of Microbiology, Immunology, and Infection (2015), http://dx.doi.org/ 10.1016/j.jmii.2015.08.014

7. Liao C, Lai C, Hsu M, Huang Y, Chu F, Hsu H, et al. Correlation between time to positivity of blood cultures with clinical presentation and outcomes in patients with Klebsiella pneumoniae bacteremia: Prospective cohort study. Clin Microbiol Infect, 2009; 15: 1119-1125.

8. Peralta G, Roitz M, Sanchez M, Garrido J, Ceballos B, Rodriguez-Lera $\mathrm{M}$, et al. Time to positivity in patients with Escherichia coli bacteremia. Clin Microbiol Infect, 2007; 13: 1077-82.

9. Peralta G, Rodriguez-Lera M, Garrido J, Ansorena L, Roiz P. Time to positivity in blood cultures of adults with Streptococcus pneumoniae bacteremia. BMC Infect Dis, 2006; 6:79

10. Pavic M, Bronic A, Kopcinovic LM. Procalcitonin in systemic and localized bacterial infection. Biochemia Medica, 2010; 20(2): 236-41.

11. Guo S, Zhou Y, Hu Q, Yao J, Wang H. Procalcitonin is a marker of Gram-negative bacteremia in patients with sepsis. The American Journal of the Medical Sciences, 2015; 349: 499-504.

12. Meisner M. Procalcitonin-biochemistry and clinical diagnosis. $1^{\text {st }}$ Ed., Bremen, UNI-MED, 2010; 35-36

13. Gopi A, Ravikumar K, Ambarish M, Shwethalatha N, Shree $S$, Ashwini $K$, et al. Time to positivity of microorganism with BACTEC 9050:- An 18-month study among children of 28 days to 60 months in a South Indian Tertiary Hospital. Int J Microbiol Res, 2011; 2(1): 12-7.

14. Shah SS, Downes KJ, Elliott MR, Bell LM, McGowan KL, Metlay JP. How long does it take to "rule out"bacteremia in children with central venous catheters?. American Academy of Pediatrics, 2008; 121: $135-41$
15. Alfouzan W, Azizieh F, Dhar R. Correlation between inoculum volume, positivity rates, and microorganisms isolated from blood cultures. African Journal of Microbiology Research, 2014; 8(28): 27052709.

16. Dolma K, Gautam D, Devi K, Singh T. A comparative study on the efficacy of first vs. second blood culture set in the diagnosis of bacteremia in Central Referral Hospital, Sikkim, India. International Journal of Medical Research and Review, 2016; 4(7): 1243-1249.

17. Abe R, Oda S, Sadahiro T, Nakamura M, Hirayama Y, Tateishi $Y$, et al. Gram-negative bacteremia induces greater magnitude of inflammatory response than Gram-positive bacteremia. Critical Care, 2010; 14(2):R27. doi: $10.1186 /$ cc8898.

18. Asaad A. Coagulase-negative Staphylococci clinical isolates: infectious or contaminant, that is the question. Int J Clin Med Microbiol, 2016; 1 : 102. doi: https://doi.org/10.15344/2456-4028/2016/102.

19. Becker K, Heilmann C, Peters G. Coagulase-negative Staphylococci. Clinical Microbiology Reviews, 2014; 27: 870-926.

20. Savithri $M$, Iyer $V$, Jones $M$, Yarwood $T$, Looke $D$, Kruger $\mathrm{P}$, et al. Epidemiology and significance of coagulase-negative staphylococci isolated in blood cultures from critically ill adult patients. Crit Care Resusc, 2011; 13: 103-7.

21. McGowan K, Foster J, Coffin S. Out patient pediatric blood cultures: Time to positivity. Pediatrics, 2000; 106 (2 Pt 1):251-5.

22. Ruimy R, Lefevre L, Andremont A. Short time to positivity in blood culture with clustered Gram-positive cocci on direct smear examination is highly predictive of Staphylococcus aureus. Am J Infect Control, 2005; 33: 304-6.

23. Garcia-Vazquez E, Fernandez-Rufete $A$, Hernandez-Torres A, Canteras M, Ruiz J, Gomez J. When is coagulase-negative Staphylococcus bacteremia clinically significant?. Scandinavian Journal of Infectious Diseases, 2013; 45: 664-671.

24. Kassis C, Rangaraj G, Jiang Y, Hachem RRI. Differentiating culture samples representing coagulase-negative staphylococcal bacteremia from those representing contamination by use of time to positivity and quantitative blood culture methods. Journal of Clinical Microbiology, 2009; 47(10): 3255-3260.

25. Leli C, Ferranti M, Moretti A, Dhahab ZSA, Cenci E, Mencacci A. Procalcitonin levels in Gram-positive, Gram-negative, and fungal bloodstream. Disease Markers, 2015; 2015: 1-8.

26. Charles E, Ladoire S, Aho S, Quenot J, Doise J, Prin S, et al. Serum procalcitonin elevation in critically ill patients at the onset of bacteremia caused by either Gram-negative or Gram-positive bacteria. BMC Infect Dis, 2008; 8:38.

27. Wanatabe Y, Oikawa N, Hariu M, Fuke R, Seki M. Ability of procalcitonin to diagnose bacterial infection and bacteria types compared with blood culture findings. 
International Journal of General Medicine, 2016; 9: 325-331.

28. Li S, Rong H, Guo Q, Chen Y, Zhang G, Yang J. Serum procalcitonin levels distinguish Gram-negative bacterial sepsis from Gram-positive bacterial and fungal sepsis. J Res Med Sci. 2016; 21:39

29. Kumar S, Ingle H, Prasad DVR, Kumar H. Recognition of bacterial infection by innate immune sensors. Critical Reviews in Microbiology, 2013;39(3):229-246.

30. Yan S, Sun L, Jia H, Gao W, Yang J, Zhang G. Procalcitonin levels in bloodstream infections caused by different sources and species of bacteria. American Journal of Emergency Medicine. 2017; 35: 579-583.

31. Nieuwkoop C, Bonten T, Wout J, Kuijper E, Groeneveld $\mathrm{G}$, Becker $\mathrm{M}$, et al. Procalcitonin reflects bacteremia and bacterial load in urosepsis syndrome: A prospective observational study. Critical Care, 2010; 14(6): R206.

32. Schuetz P, Mueller B, Trampuz A. Serum procalcitonin for discrimination of blood contamination from bloodstream infection due to coagulase-negative staphylococci. Infection, 2007; 35: 352-355. 